\title{
A hybrid control strategy for force and precise end effector positioning of a twisted string actuator
}

\author{
Andres San-Millan, Mohssen Hosseini and Jamie Paik *†
}

December 22, 2020

\begin{abstract}
We propose a new control approach for the twisted string actuation (TSA) system; a hybrid control scheme consisting of two nested loops, considering the motor angle and the axial force at the clamping point of the actuator as the system outputs. We show that measuring the axial force at the base of the actuator, we can effectively estimate and compensate the coupling torque between the motor and the external load. Then, compensation of the coupling torque allows formulating a linear control law to control the angular position of the actuator and, at the same time, implement an external control loop to regulate the force applied by the actuator. Simulated and experimental results validate the proposed original control scheme. Furthermore we highlight the contributions of this work in the broader context of control of twisted string actuators.
\end{abstract}

${ }^{*}$ This work was supported by the National Centre of Competence in Research (NCCR) in Robotics (Switzerland).

${ }^{\dagger}$ A. San-Millan, M. Hosseini, and J. Paik work at the École polytechnique Fédérale de Lausanne, Lausanne 1015, Switzerland (e-mail: $\quad$ andres.sanmillanrodriguez@epfl.ch; mohssen.hosseini@epfl.ch; jamie.paik@epfl.ch).

\section{Introduction}

Twisted string actuator (TSA) systems enable linear transmission with high-performance in a compact form factor [34, 13]. Using a proper combination of string parameters including radius and total length, and electrical motor specifications, it is possible to generate linear motions with high pulling forces from low-torque rotary motors [22], thus satisfying a wide range of requirements for output speed and force of highlyintegrated mechatronic devices.

The high transmission ratio of TSAs is attractive for robotic systems $[14,35]$, especially for applications where energy-efficient and costeffective solutions are required, such as robot manipulators [27, 25] and grippers, anthropomorphic hands [15, 22, 20, 34], and mobile robots [37], they provide lightweight and highly customizable solutions. Additionally, TSAs have been used successfully in functions that require compact and mechanically simple transmission with some level of compliance; among these, soft robotics [8] and human-machine interaction (HMI) devices like wearable robots and exoskeletons [36, 4] or haptic devices [11, 33, 10, 26].

However, the main drawback of TSAs is the highly nonlinear behavior of the transmission ratio produced by the twisting of the string. The 
mathematical (kinetostatic) models of a twisted string transmission system presented in [7, 23], showed that there is a variable transmission relation between the angular position of the motor and the contraction produced in the longitudinal axis of the actuator. There are some mechanical solutions for linearizing the input-output relationship of the system $[20,18]$; however, optimal control of the TSA while producing stable behavior is still an important topic under study $[23,24,33,16,31,38]$ due to several challenges.

TSAs are based on the well-known principle that twisting a string by an electric motor produces a contraction the length of the string thereby leading to a linear displacement at the other end. The dynamic model of the TSA can be divided into two submodels, the first, represents the motor dynamics and the second, describes the behavior of the string. The coupling torque the string exerts on the motor link the submodels. Most state-of-the-art research models the electric motor as a permanent magnet direct current motor (PMDCM) having linear dynamics, while the string is often modeled as a nonlinear kinetostatic form, where the inertia of the string is neglected with respect to that of the motor $[7,23]$. The saturation of the motor is ignored in this classical approach, thus lowering the performance of the closed-loop scheme. Still, it is important to note that due to the small, generalized reduction ratio of the TSA [23], high angular displacements produce small linear displacements and in order to achieve significant linear motion, the motor has to operate at maximum speed most of the time, i.e. at the saturation voltage.

The state-of-the-art literature reinforces that current control schemes for TSAs do not take into account the saturation of the motor, leading to poor performance in terms of bandwidth and consequently, less stable behavior [33]. To the best of our knowledge, the maximum achievable bandwidth from TSAs has not been formally addressed so far; although experimental results of many studies [23, 31] performed position tracking for frequencies as low as $0.5 \mathrm{~Hz}$ and $0.1 \mathrm{~Hz}$ for small angular displacements. These results illustrate that with the current control schemes for TSAs, the integral actions lead to saturation of the motor and to avoid this, only slow reference signals with small amplitude can be tracked.

The main goal of this work is to demonstrate that application of a two-degrees-of freedom (2DoF) controller, combined with a linear feedback back-calculation anti-windup technique, henceforth named a 2-DoF+AW controller, to control the angular position of the motor will lead to a drastic improvement in closed-loop performance in terms of bandwidth and relative stability. Additionally, we go on to combine this position controller with an impedance controller to demonstrate suitability for integration into a robotic haptic device. The impedance controller should guarantee safe human-machine interaction by allowing adjusting the degree of compliance of the system under external perturbations, such as forces applied by the human operator [39]. The 2-DoF+AW controller design evolved from previous work on flexible robots that use simple 2-DoF controllers [6,3], which demonstrated good performance coupled with ease of design and implementation by means of simple pole placement [32].

The main contributions of this work are:

- the analytical expression of the stability condition for the simple 2-DoF controller in the presence of saturation of the actuator is derived. It is important to note that this expression is crucial as it imposes a restriction 
on the feasibility of the designed regulator. To the best of our knowledge, this restriction has never been derived or used hitherto in the design of such a regulator. Usually the regulator is constructed along the lines of a linear system and aiming to prevent saturation by means of low pass filters or by using fourth order trajectories with small accelerations [3].

- the analysis of the relative stability and performance of the 2-DoF $+\mathrm{AW}$ controller for different values of the anti-windup gain, leading to the analytical expression of the optimal value of the anti-windup gain in terms of speed of response. This expression, not previously reported, is key to the creation of a controller that produces optimal response in terms of rise time.

- we show that the idea of compensating the coupling torque adopted from the field of flexible manipulators [5] can be extrapolated to control TSAs by decoupling the dynamics of the motor and the string, this allows designing a controller for the angular position whose stability is independent of the string dynamics.

- the practical implementation of the proposed 2-DoF controller in combination with a secondary control loop built on top of the servo-control and designed to provide an impedance control and compensate the nonlinear relationship between the angle of the motor and the linear position of the end effector. This also allows us to design the parameters of a virtual mass-spring-damper (MSD) equivalent system, tailored according to desired goals, such as providing the desired rigidity to a robotic haptic device.
The paper is organized as follows. Section 2 formally states the problem and presents the mathematical kinetostatic models of the TSA transmission system. We then detail the proposed control scheme in Section 3. The stability analysis and a comparative performance improvement analysis to the traditional 2-DoF controller is included in Section 4, the experimental setup in Section 6, where simulated and realtime experimental results validate the proposed control scheme. Section 7 concludes the paper.

\section{A TSA MODEL AND PROBLEM FORMULA- TION}

As depicted schematically in Fig.1, the working principle of the TSA is length reduction by twisting a string composed of two or more strands due to geometrical constraints. The strands are connected in parallel to the motor's shaft on one end and to a linearly moving end effector on the other end.

\subsection{Kinetostatic Model}

The kinetostatic model relates the change in the angular rotation of the shaft, and the total length of the string, $p$. If the shaft is rotated by an angle, $\theta$, this rotation forces the strands into a helical shape as shown in Fig 1; thereby reducing the total length of the string.

Considering that the string is composed of $n$ parallel strands of radius, $r_{s}$, the radius of the string $r$ is given by:

$$
r_{c}=\frac{r_{s}}{\sin (\pi / n)}
$$




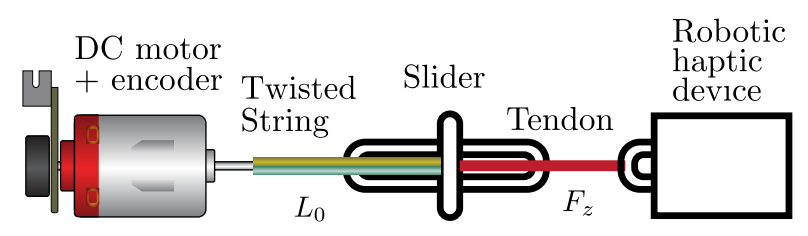

(a)

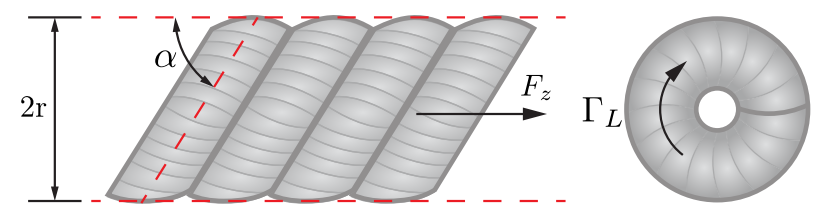

(b)

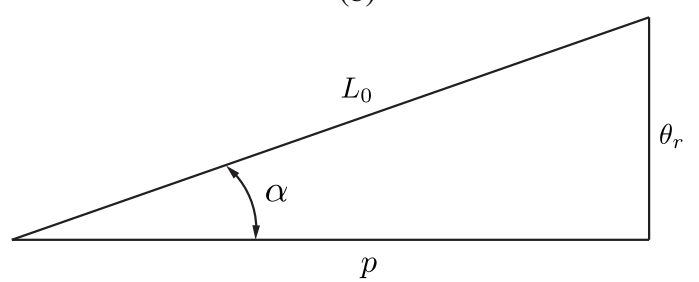

(c)

Figure 1: Schematic representation of the system. (a) Diagram of the complete TSA. (b) Lateral and axial view of the helix formed by the two strands (c) Unwrapped representation of a single strand

When the helix is closely wound, the maximum value achievable by the helix slope $\alpha$ is given by:

$$
\alpha_{\max }=\arctan \left(\frac{\pi}{\sin (\pi / n) n}\right)
$$

According to the model proposed in [23, 7], assuming a string with length $L_{0}$ and possessing infinite stiffness; the helix slope, $\alpha$, and the total length, $p$, will depend on the twisting angle, $\theta$, produced by the motor. The geometry of the helix leads to the following relations:

$$
\begin{gathered}
\alpha=\arccos \left(\frac{\sqrt{L_{0}^{2}-\theta^{2} r^{2}}}{L_{0}}\right), \\
p=\sqrt{L_{0}^{2}-\theta^{2} r^{2}},
\end{gathered}
$$

Following the naming convention stated in [23], the "generalized reduction ratio" of the TSA is given by:

$$
h(\theta)=\frac{\theta r^{2}}{p} .
$$

It is important to note that due to the restriction imposed by (2), there are also restrictions on the maximum value of $\left|\theta_{\max }\right|$ and $p_{\min }$ :

$$
\begin{gathered}
\left|\theta_{\text {max }}\right|=\frac{L_{0}}{\sqrt{r^{2}+\frac{r_{s}^{2} n^{2}}{\pi^{2}}}}, \\
p_{\text {min }}=\frac{L_{0}}{\sqrt{\frac{\pi^{2} r^{2}}{r_{s}^{2} n^{2}}+1}},
\end{gathered}
$$

where the absolute value of $\left|\theta_{\max }\right|$ accounts for the sign of $\theta$, which depends on whether the motor is turning clockwise or anti-clockwise. It should be pointed out that these limits are not "hard" limits in the sense that bigger values of $\alpha$ and smaller values of $p$ can be physically reached - but in that case, the previous equations will not describe the behavior of the system. This situation is commonly known as "overtwisting".

\subsection{Dynamic TSA Model}

In order to model the dynamics of the TSA we adopt the following customary simplifications $[16,17,14,23]$ : a) The dynamics of the string are negligible when compared to the dynamics of the motor and its behavior can be reproduced with the aforementioned kinetostatic model. b) The 
TSA is uni-directional and can only exert compressive forces. Therefore, the string can only return to its original length if there is a external tensile force. c) The acceleration and speed of the end effector of the TSA are small enough so that the resultant torques in the motor are negligible when compared to the torques produced by the external forces applied to the end effector.

Considering that the string is twisted by a DC motor, the complete dynamics of the system can be described using a Hammerstein-Wiener model. In this model, the linear dynamics of the motor are placed between the static nonlinear saturation of the motor and the static nonlinear behavior of the string. It is noteworthy that the static nonlinear relation between angular position and displacement provided by the string is given by (4).

The dynamic equilibrium in the output shaft of the motor is given as:

$\Gamma_{m}(t)=K_{m} I(t)=J_{0} \ddot{\theta}(t)+\nu \dot{\theta}(t)+\Gamma_{p}(t)+\Gamma_{L}(t)$,

where $J_{0}$ is the rotor inertia, $\nu$ is the viscous friction coefficient, $\Gamma_{p}$ is a perturbation to the system that comprises the effects of any nonlinearity, which dominates any linear effect for small positioning errors, such as the friction at the slider of the TSA or the dry friction at the shaft of the motor. Then, $\Gamma_{m}$ is the torque applied by the motor, $K_{m}$ is the electromechanical constant of the motor that relates the current, $I$, to the torque in the motor side, and $\Gamma_{L}$ is the external torque produced by the string. Considering that the motor is endowed with linear amplifiers with dynamics significantly faster than the mechanical dynamics, the current, $I$ can be made proportional to a control voltage $V$, leading to the following expression:

$$
\hat{K}_{m} V(t)=J_{0} \ddot{\theta}(t)+\nu \dot{\theta}(t)+\Gamma_{p}(t)+\Gamma_{L}(t),
$$

The external torque, $\Gamma_{L}$, can be related to any external axial force $F_{z}$ applied to the end effector of the TSA by using the following expression:

$$
\Gamma_{L}(t)=F_{z}(t) h(\theta),
$$

and thus (8) leads to:

$$
\hat{K}_{m} V(t)=J_{0} \ddot{\theta}(t)+\nu \dot{\theta}(t)+\Gamma_{p}(t)+F_{z}(t) h(\theta) .
$$

\subsection{Problem Formulation}

The problem that is tackled in this paper is to control the linear displacement of the end effector, $p$, by using the input voltage to the motor, $V$, as the only control input to the system. This task is challenging from both a practical engineering point of view and from the perspective of control theory. On the one hand, it is not practically possible to directly measure the position of the end effector while maintaining the TSA's design compactness. Unlike rotational encoders, where the device is static and can be integrated into the body of the motor, accurate measurements of the position of the end effector require the utilization of bulky sensor systems such as linear potentiometers that span the entire range of displacement of the TSA [17], or expensive laser-based measuring systems. On the other hand, from the control theory perspective, there are many important challenges: a) The axial force applied to the end effector of the TSA and the equivalent torque applied to the motor have a strong nonlinear dependency on the angular position of the motor given by $(5)$, b) The 
saturation of the control signal is determined by the maximum voltage admissible by the motor and, c) As there is no direct measurement of the position of the end effector, its actual value cannot be used to close the control loop.

\section{CONTROL ALGORITHM}

As stated in the Introduction, the TSA under consideration will be used in a robotic haptic device for a virtual reality environment. Therefore, to guarantee a safe human-machine interaction between the device and the user, it is necessary to implement an impedance controller where the degree of compliance can be modulated by setting the parameters of the virtual MSD system, i.e., the displacement of the end effector of the device will react linearly to the external forces applied by the user of such an MSD system. It is important to note that this approach differs from [24], as it considers the inertia of the MSD system and the force applied by the virtual spring is not proportional to the elongation of the initial length of the string but to its elongation to a desired set point specified by the user. This implementation allows greater flexibility and fidelity when mimicking an MSD system.

As the impedance control relies on the accurate control of the position of the end effector, the entire control scheme consists of two nested controllers designed independently. The innerloop controller is based on linear force feedback aimed at decoupling the motor and string dynamics, thus allowing design of a 2-DoF controller independently depending on motor dynamics and control specifications. This technique has been successfully applied in previous work on flexible robots $[6,3]$. The outer-loop controller provides force control of the end effec- tor, compensating for the nonlinear relationship introduced by the string of the TSA. This section describes the utilized controllers and demonstrates the stability of the overall control scheme.

\subsection{Inner Control Loop}

It is well-known that the effects of friction in control of dc motors can be minimized using highgain linear feedback. It was demonstrated in [29] that the 2-DoF controller employed herewith is highly insensitive to friction and that the sensitivity ratio to $V$, can be reduced arbitrarily by increasing the gains of the controller. Therefore, a reduced order model of (11) can be used, where $\Gamma_{p}(t)$ is negligible when compared to $F_{z}(t) h(\theta)$, thus leading to:

$$
\hat{K}_{m} V(t)=J_{0} \ddot{\theta}(t)+\nu \dot{\theta}(t)+F_{z}(t) h(\theta) .
$$

Assuming that the external axial force applied to the system is $F_{z}(t)$ and that the generalized reduction ratio $h(\theta)$ can be estimated or measured, we define the following control signal $V_{c}(t)$

$$
V_{c}(t)=V(t)-\frac{\hat{F}_{z}(t) \hat{h}(\theta)}{\hat{K}_{m}}
$$

where $\hat{F}_{z}(t)$ and $\hat{h}(\theta)$ are the estimated or measured values of $F_{z}(t)$ and $h(\theta)$, respectively. Substituting the above equation in (11) yields:

$$
\hat{K}_{m} V_{c}(t)=J_{0} \ddot{\theta}(t)+\nu \dot{\theta}(t)
$$

where the coupling torque produced by the external axial load is compensated, and thus the resulting motor dynamics can be written in the Laplace domain as:

$$
\frac{\theta(s)}{V_{c}(s)}=M(s)=\frac{A}{s(s+B)}
$$


with $A=\hat{K}_{m} / J_{0}$, and $B=\nu / J_{0}$. Therefore, the controller $R_{i}(s)$ can be designed independently of the TSA's string since the string dynamics are decoupled.

The design of the inner controller $R_{i}(s)$ is based on the structure of the 2-DoF controller [21]. The two nested control loops shown in Fig.2 allow for arbitrary pole placement in closed-loop when the two transfer functions for controllers $C_{1}(s)$ and $C_{2}(s)$ are properly designed [3]. The closed-loop transfer function between the motor position, $\theta$ and the motor reference, $\theta^{*}$, is given by:

$$
\theta(s)=\frac{C_{1}(s) A}{s^{2}+s B+A\left(C_{1}(s)+C_{2}(s)\right)} \theta^{*}(s)
$$

where the following equalities hold:

$$
\begin{gathered}
C_{1}(s)=\frac{a_{2} s^{2}+a_{1} s+a_{0}}{s(s+g)}, \\
C_{2}(s)=\frac{b_{1} s+b_{0}}{(s+g)} .
\end{gathered}
$$

As can be seen in $[6,3]$, the closed-loop transfer function $G_{C L}$, which relates the angular position of the motor and the references $\left(\theta(s) / \theta^{*}(s)\right)$, is usually designed such that it is composed of 4 poles and 2 zeros. In order to maximize the closed-loop bandwidth of the controlled motor and to provide the fastest response to changes in the reference signal, these poles and zeros are placed such that the resulting system is a secondorder critically-damped system. To achieve this pole-zero placement, two of the poles are used to cancel the two zeros and the remaining two poles are coincident and placed on the real axis as follows:

$$
G_{C L}(s)=\frac{p_{2}^{2}\left(s+p_{1}\right)^{2}}{\left(s+p_{1}\right)^{2}\left(s+p_{2}\right)^{2}} .
$$

Substituting (16) and (17) into (15), the following expression is obtained:

$G_{C L}(s)=\frac{A\left(a_{2} s^{2}+a_{1} s+a_{0}\right)}{\left(\begin{array}{l}s^{4}+(B+g) s^{3}+\left(A\left(b_{1}+a_{2}\right)+B g\right) s^{2} \\ +\left(b_{0}+a_{1}\right) A s+A a_{0}\end{array}\right)}$

where it can be seen that combining expressions (16) to (19), the following design rules can be derived:

$$
\begin{aligned}
& a_{2}=\frac{p_{2}^{2}}{A}, \quad a_{1}=\frac{2 p_{1} p_{2}^{2}}{A}, \quad a_{0}=\frac{p_{1}^{2} p_{2}^{2}}{A} \\
& b_{1}=\frac{p_{1}^{2}+4 p_{1} p_{2}+B^{2}-2 B\left(p_{2}+p_{1}\right)}{A} \\
& b_{0}=\frac{2 p_{1}^{2} p_{2}}{A}, \quad g=2\left(p_{2}+p_{1}\right)-B .
\end{aligned}
$$

The block diagram of the classical design of the 2-DoF controller can be seen in Fig.2. It is important to note that the positions of $p_{1}$ and $p_{2}$ must be placed as far away as possible from the imaginary axis to lead to high gain values in the controller and, so that the reduced order model (12) holds. This is common practice in previous works such as [3], however these high gain values and the presence of the integral action in $C_{1}(s)$ make the resulting control scheme very sensitive to the presence of saturation of the actuator, thus leading to the integrator windup.

This problem has been addressed in the past by using smooth and slow reference signals so that the actuator never reaches saturation, however in the case of the TSA, the string leads to a high reduction ratio, i.e. to achieve a small linear displacement it is necessary to produce a large angular displacement, thus if we want to produce significant linear displacements, the motor will have to operate most of the time in the 
saturation zone. This is why the analysis and application of the anti-windup technique to the 2-DoF controller is of utmost interest for the case of a TSA.

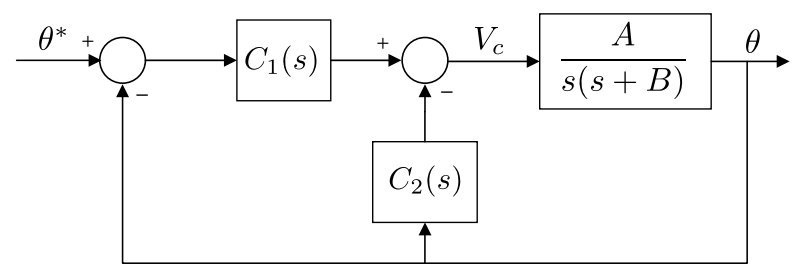

Figure 2: Proposed inner control loop. Block diagram of the 2-DoF controller

In order to take an advantage of the classical 2-DoF controller, which is robust with good disturbance rejection and easy to design, and to solve the problem of the saturation of the integral action, the following linear feedback backcalculation anti-windup technique [1] can be implemented as shown in Fig.3, where the saturation block is a mathematical model of the actual saturation observed in the motor, and the antiwindup term $C_{3}(s)$ holds the following equality:

$$
C_{3}(s)=\frac{K_{A W} \cdot a_{0}}{g s},
$$

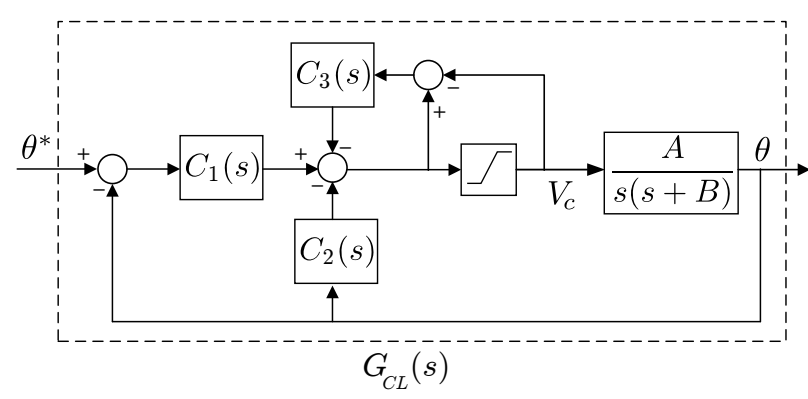

Figure 3: Inner control loop. 2-DoF+AW controller
Usually, in the case of PID controllers the gain $K_{A W}$, is chosen using a rule of thumb based on the integral and derivative time constants, $T_{i}$ and $T_{d}$, so that $K_{A W}=1 / \sqrt{T_{i} T_{d}}$ [1], but in this case there are no such time constants readily available and, what is more, the aforementioned rule of thumb does not produce an optimal response. Therefore, the main goal of this paper is to develop an analytical rule for the design of the term $K_{A W}$, so that the response to a step signal is optimal in terms of rise time (which is minimized), whereas mimicking a second-order critically damped system. This contribution is relevant because it simplifies the design procedure of the $2-\mathrm{DoF}$ and solves the abovementioned windup problem [3].

\subsection{Outer Control Loop}

Let us denote $G_{C L}(s)$ as the transfer function that relates $\theta(s) / \theta^{*}(s)$. Provided that $L_{0}$ and $r$ are known, the nonlinear relationship provided by (4) can be used to transform the linear position of the end effector $p^{*}(t)$ to a desired setpoint of angular position $\theta^{*}(t)$ as follows:

$$
\theta^{*}(t)=\sqrt{\frac{L_{0}^{2}-p^{*}(t)^{2}}{r^{2}}}
$$

This relationship can be used to compensate the effect of the nonlinear relationship between the angular position and the linear position, thereby allowing control of the former using the inner control loop. To provide compliance to the whole system, the measurement of the external axial force applied $F_{z}$, is added to the set-point of linear position $P^{*}$ to produce the desired reference linear displacement $p^{*}$. The transfer function that relates the external force and the linear displacement of the TSA is designed so that it mimics the MSD system dynamics, where the 
mass, $m$, the spring constant, $k$, and the damping coefficient, $b$, can be designed independently. This results in the following equivalent transfer function:

$$
C_{4}(s)=\frac{1 / m}{s^{2}+s(b / m)+k / m}
$$

The impedance controller is depicted in Fig. 4. It can be seen that by changing the position of the poles of (23), the dynamic response of the system can be adjusted in order to obtain different values of stiffness and damping. However, it should be noted that because of the bandwidth of the closed-loop response of the system, determined by the location of the dominant poles of (18), the natural frequency of the virtual spring produced by the impedance controller (23) cannot be higher than the bandwidth of the closedloop response. The complete control scheme is shown in Fig. 5.

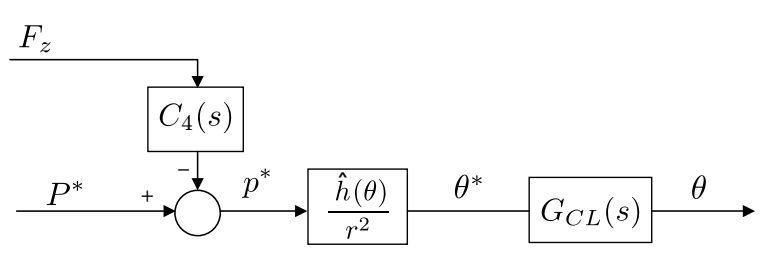

Figure 4: Outer controller. Impedance control of the TSA

\section{Stability analysis}

Since the outer-loop controller is composed of a feedforward implementation, the stability of the whole control scheme is determined by the closed-loop stability of the position servocontrol, i.e., the stability of the $2-\mathrm{DoF}+\mathrm{AW}$ controller. Additionally, as stated earlier, the bandwidth of the position servo-control will determine the maximum frequency of the virtual spring. As a consequence, this section is devoted to the analysis of the proposed 2-DoF+AW controller.

If the transfer function of the tracking controller (16) is expressed in the standard noninteracting form of a PID as follows:

$$
C_{1}(s)=K+\frac{K}{s T_{i}}+\frac{s K T_{d}}{1+\frac{s T_{d}}{N}},
$$

where $K$ is the proportional gain, $T_{i}$ and $T_{d}$ are the integral and derivative time constants respectively, and $N$ is a high frequency gain used to ensure that the transfer function of the derivative action is proper. Then, the $2-\mathrm{DoF}+\mathrm{AW}$ controller can be expressed in state-space form [30] as follows:

$$
\begin{aligned}
\frac{d x}{d t}= & \quad(F-Q H) x+\left(G-Q-Q D_{r}\right) \theta^{*} \\
U= & H x+D_{r} \theta^{*}-D \theta+Q V_{c} \\
V_{c}= & \operatorname{sat}(U)
\end{aligned}
$$

where the values of the aforementioned vectors and matrices for the proposed controller take the following expressions, which correspond to the observer approach, [2]:

$$
\begin{aligned}
F=\left[\begin{array}{ccc}
0 & 0 & 0 \\
0 & \frac{-N}{T_{d}} & 0 \\
0 & 0 & -g
\end{array}\right], \quad G_{r}=\left[\begin{array}{c}
\frac{K}{T_{i}} \\
\frac{N}{T_{d}} \\
0
\end{array}\right], G=\left[\begin{array}{c}
\frac{K}{T_{i}} \\
\frac{N}{T_{d}} \\
b_{0}-b_{1} g
\end{array}\right] \\
H=\left[\begin{array}{lll}
1 & -K N & 1
\end{array}\right], \quad D_{r}=K(1+N), \\
Q=\left[\begin{array}{lll}
\frac{K_{A W} K}{T_{i}} & 0 & 0
\end{array}\right]^{T}, \quad D=K(1+N)+b_{1}
\end{aligned}
$$




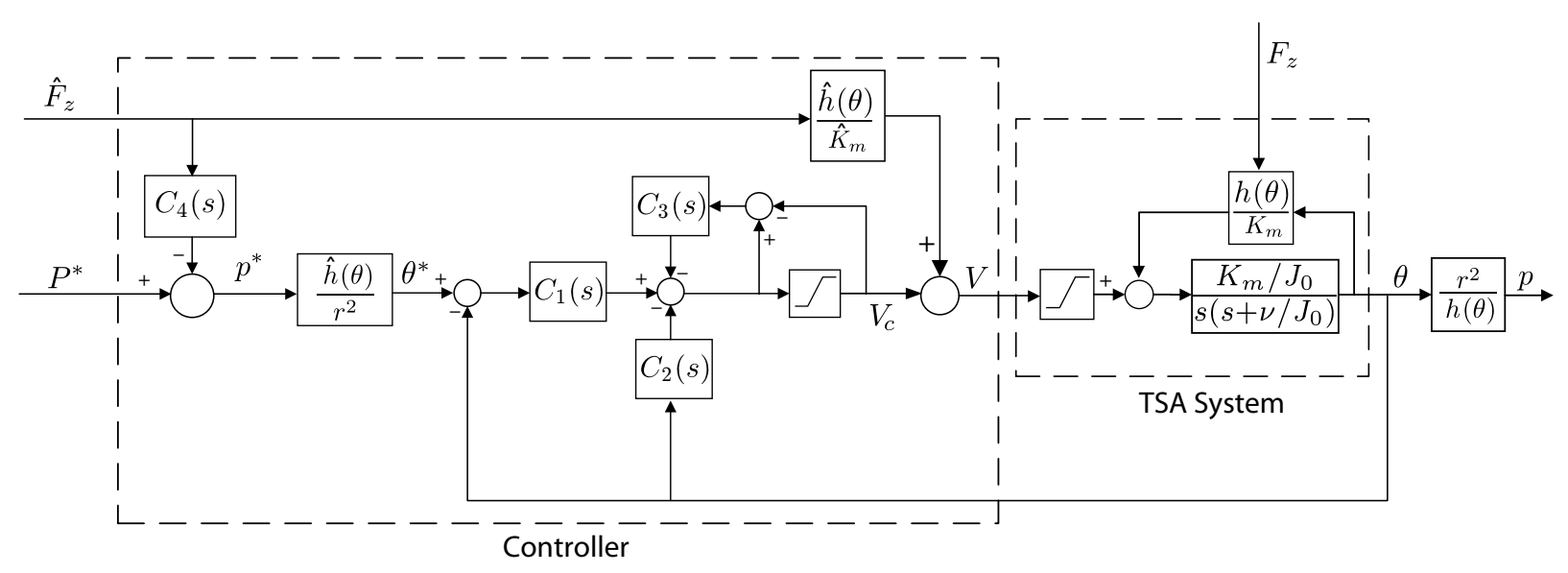

Figure 5: The position and impedance control scheme of the TSA system

As stated in [30], a system with a saturating actuator can always be reduced to the standard Lur'e system configuration with a linear system having a nonlinear feedback. For timeinvariant monotonic linearities, such as the saturation, sufficient conditions for stability can be obtained from the off-axis circle criterion [19]. Considering that the nonlinear feedback element is saturation, the linear system $G^{*}(s)$ is given by:

$$
G^{*}=-\frac{G_{c} M-W}{1+W}
$$

where $M$ is the transfer function of the DC motor, and $G_{c}$ and $W$ are given by the following expressions:

$$
\begin{aligned}
& W=H(s I-F)^{-1} Q, \\
& G_{c}=H(s I-F)^{-1} G+D
\end{aligned}
$$

Theorem 1. If the linear system $G^{*}(s)$ has all poles in the closed left half-plane and nonlinear feedback from a saturation, the closed-loop is absolutely stable provided that a straight line through the origin can be given a nonzero slope, such that $G^{*}(i \omega)+1$ is strictly to the right of the line. $G^{*}(i \omega)+1$ will be strictly positive real $(S P R)$ if it is situated to the right of the imaginary axis.

Proof. The proof can be found in [19], p. 169.

It is important to note that this theorem is different from Popov's criterion [28] because $G^{*}(s)$ does not need to be asymptotically stable, i.e. it can present a pole in the origin and because the geometric criterion for the asymptotic stability is that $G^{*}(i \omega)+1$ lies to the right of a line with a slope $q \neq 0$ and is not restricted to the right side of a vertical line, as in Popov's criterion [40].

The linear system $G^{*}(s)$ for the proposed control scheme presents the following numerator and denominator:

$$
\begin{aligned}
G_{n u m}^{*}= & K_{A W} T_{d} K s^{4} \\
& +\left[\begin{array}{c}
K_{A W} K\left(N+T_{d}(B+g)\right. \\
-T_{i} T_{d} A\left(K(N+1)+b_{1}\right)
\end{array}\right] s^{3}
\end{aligned}
$$




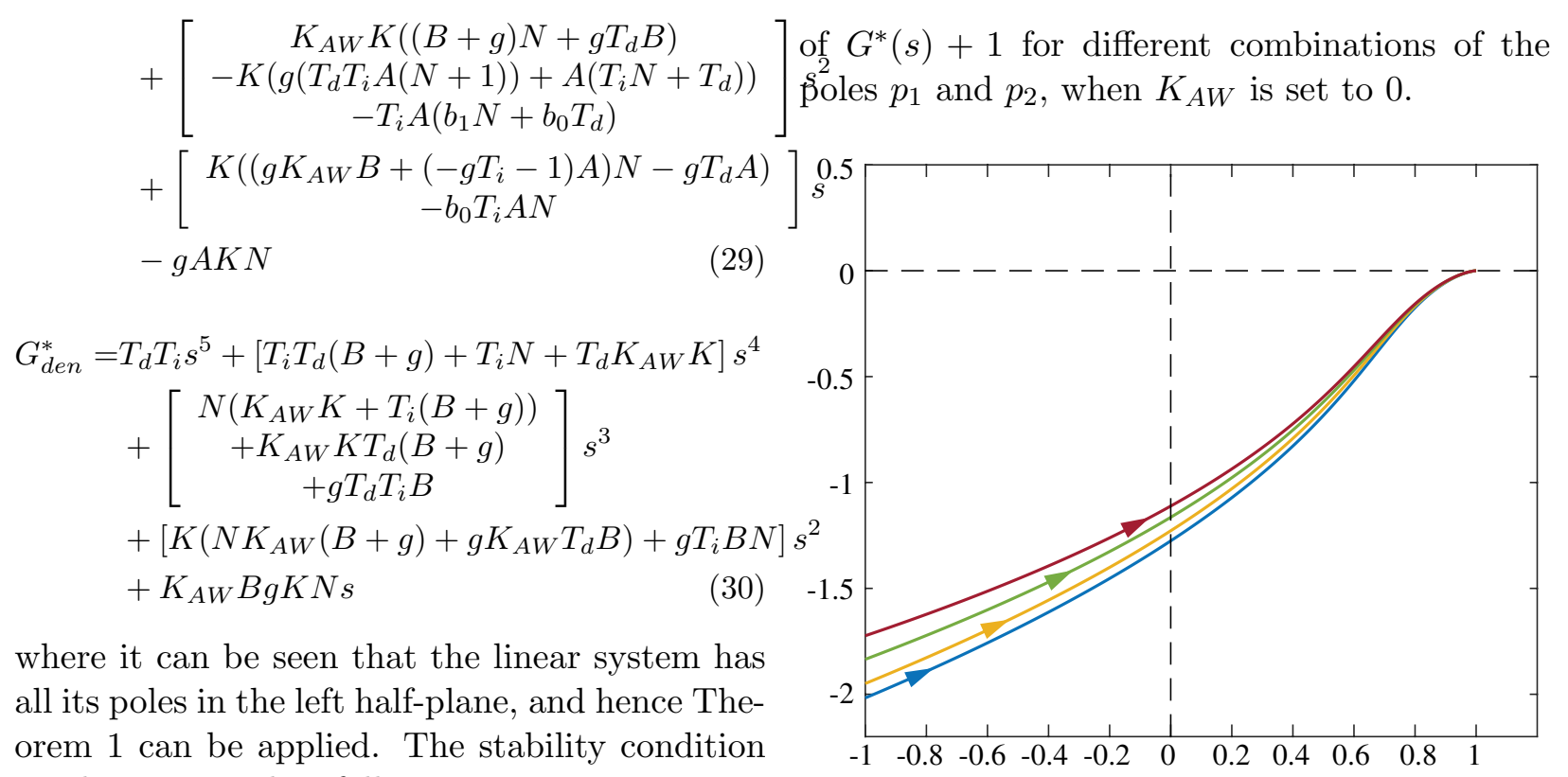
can be expressed as follows:

If there exists a constant $q \neq 0$ such that for all $\omega \geq 0$

$$
q \Re\left[G^{*}(i \omega)+1\right]-\Im\left[G^{*}(i \omega)+1\right]>0
$$

then the closed-loop system is asymptotically stable. Numerical evaluation of the expression (31) allows determining if the combination of the controller and the anti-windup gain leads to a stable controller.

In this paper, we focus on analyzing two scenarios regarding the evaluation of expression (31).

$C$ ase 1: The traditional design of the 2-DoF controller, i.e., when the anti-windup gain $K_{A W}$ is set to 0 . In this case the evaluation of (31) allows determining whether the system will be stable depending on the combinations of the poles $p_{1}$ and $p_{2}$. This is an important extension of the design procedure of the 2-DoF controller, which has not been taken into account in previous work $[6,3]$. Fig. 6 shows the Nyquist curves

Figure 6: Nyquist diagram of $G^{*}(i \omega)+1$ when $K_{A W}=0$ for different placement of the closedloop poles (from left to right) $p_{2}=50, p_{1}=60$; $p_{2}=40, p_{1}=50 ; p_{2}=30, p_{1}=40 ; p_{2}=20$, $p_{1}=30$

From Fig. 6, it can be seen that placing the closed-loop poles further from the imaginary axis to achieve a fast response results in Nyquist curves moving closer to the real axis at smaller values of $\omega$. This means that expression (31) is satisfied for smaller and smaller values of $q$. This figure also shows that the classical design of the 2-DoF controller without anti-windup leads to a non-SPR system for $G^{*}(i \omega)+1$.

At the limit situation when $\lim _{q \rightarrow 0}$ and $\lim _{\omega \rightarrow 0}$, equation (32) determines the necessary condition for the stability of the closed-loop system when $K_{A W}=0$.

$$
\left(T_{i}\left(g^{2} A B K+b_{0} g A B\right)-g^{2} A K\right) N^{2}>0
$$


It is important to note that expression (32) is an additional constraint that must be satisfied when designing a 2-DoF controller in order to guarantee stability in the presence of saturation of the actuator. This constraint, to the best of our knowledge has never been stated before when working with 2-DoF controllers and instead, to avoid instability, smooth and slow reference signals are used as workaround.

Case 2: The traditional design of the 2DoF controller, i.e. when the anti-windup gain $K_{A W} \neq 0$. In this case, the anti-windup action displaces the Nyquist curves of $G^{*}(s)+1$ closer to the imaginary axis for small values of $\omega$. This effect can be seen in Fig. 7, where the curves for different values of $K_{A W}$ are shown in the case of $p_{2}=50, p_{1}=60$, i.e., when the closed-loop poles are placed further from the imaginary axis and the system has a smaller relative stability.

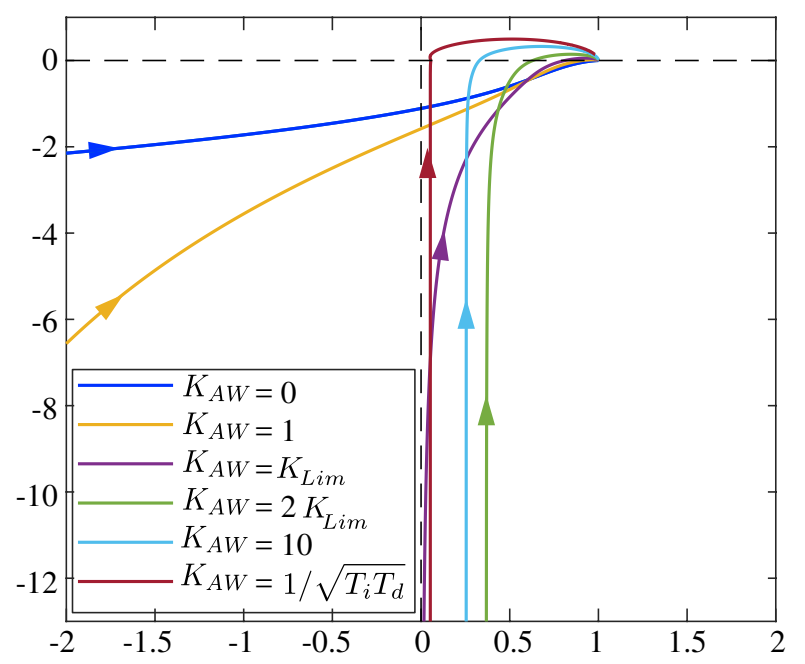

Figure 7: Nyquist diagram of $G^{*}(i \omega)+1$ in the case $p_{2}=50, p_{1}=60$ for different values of $K_{A W} \cdot K_{\text {Lim }}$ is obtained from expression (33)

The value of $K_{A W}$ that guarantees that
$G^{*}(i \omega)+1$ is SPR can be obtained from $\Re\left[G^{*}(i \omega)+1\right]=0$ when $\lim _{\omega \rightarrow 0}$, thus leading to the following expression:

$$
K_{A W} \geq \frac{g T_{i} B}{\left(T_{i} B-1\right) K g+b_{0} T_{i} B}
$$

that can be expressed in terms of the closed-loop poles and the parameters of the motor $\left(p_{1}, p_{2}\right.$, $A$, and $B$ ) as:

$K_{A W} \geq \frac{A B\left(B^{2}+4\left(2 p_{1} p_{2}-p_{1} B\right)+4\left(p_{2}^{2}+p_{1}^{2}-p_{2} B\right)\right)}{2 p_{1} p_{2}\left(2\left(p_{1}+p_{2}\right)^{2} B-\left(p_{1} p_{2}+B^{2}\right)\left(p_{1}+p_{2}\right)\right)}$

It can be seen that the combination of (34) and (20) determine the whole set of design rules needed to tune the $2-\mathrm{DoF}+\mathrm{AW}$ controller, (i.e. the inner controller of the TSA).

It is important to highlight that if condition (34) is satisfied, the closed-loop response presents no overshoot and, because it is much smaller than the expression proposed using the rule of thumb in [1], i.e. $K_{A W}=1 / \sqrt{T_{i} T_{d}}$, the rise time of the closed-loop system is faster, thus leading to an improvement in the performance. Fig. 8 shows a comparison of the evolution of the angular position of the motor tracking a step signal reference when the same controller is designed with different values of $K_{A W}$.

\section{Performance comparison with alternative algorithms}

Several control methods devoted to the control of TSA exist, [23, 18, 31, 38]. However, some are focused on the combination of two motors and tendons to solve the well-known issue of unidirectionality (specific to TSAs) [38], whereas others address the mechanical compensation of 


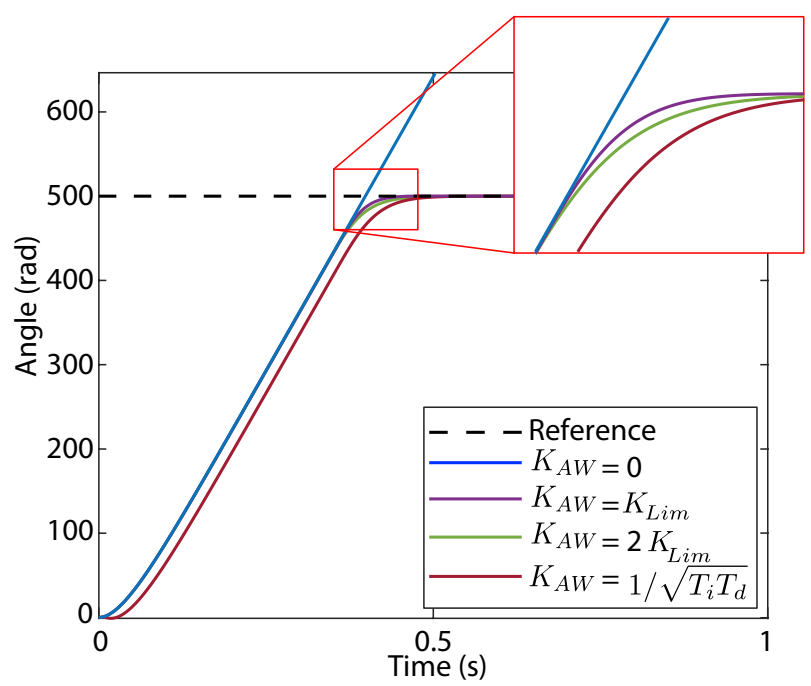

Figure 8: $G^{*}(i \omega)+1$ in the case $p_{2}=50, p_{1}=60$ for different values of $K_{A W} \cdot K_{\text {Lim }}$ is obtained from expression (34)

the nonlinear relationship between linear displacement and angular displacement [18]. Since the main contributions of this paper lie within the realms of control engineering, the performance of the proposed controller is compared to other similar strategies found in $[23,31]$.

The preceeding references illustrate the two main approaches that have been employed to address the saturation of the integral action:

On the one hand, the controller proposed in [23] pertains a second-order sliding manifold approach for a general mechanical system, which is comparable to a standard PID. It is true that while the proposed controller outperforms a standard PID, there is no consideration of the problem of saturation of the actuator and therefore, it is not able to track reference signals of high amplitude. Furthermore, the design methodology proposed in [23] is more complex than the one proposed here (given by expres- sions (20), and (34)). The control scheme in [23] delivers a response with significant overshoot (as can be seen from the results of [23]) and in addition, to the saturation of the integral action when tracking steep reference signals (thus limiting the workspace to the small frequencies and amplitudes used in [23]). These drawbacks are overcome in the proposed scheme, shown clearly in Fig. 9.

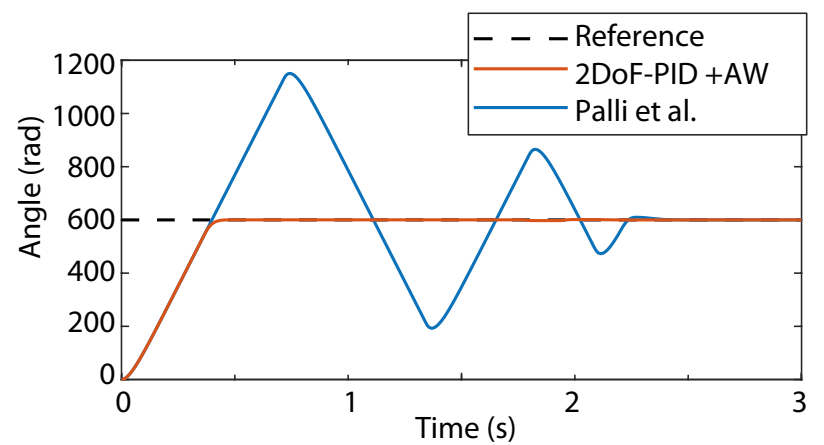

(a)

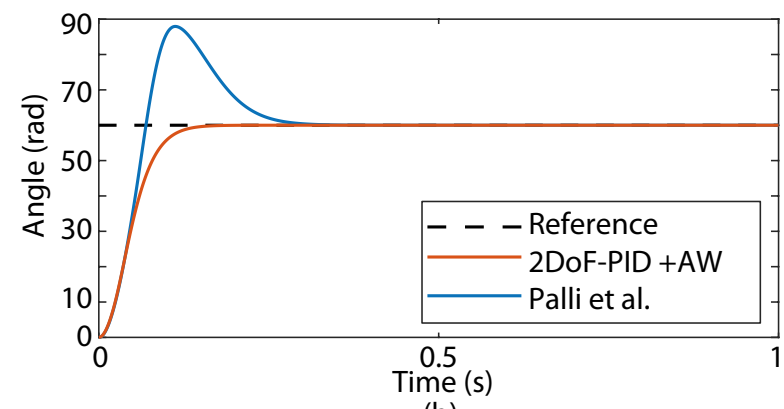

(b)

Figure 9: Closed-loop performance comparison between control methodology proposed in [23] and our suggested methodology. (a) Example of oscillations induced by saturation in the controller proposed in [23]. (b) Example of overshoot produced by the controller in [23] when tracking steep signals

On the other hand, the controller proposed in [31] concerns a disturbance observer that esti- 
mates the external force applied to the TSA to, then obtain an equivalent torque and compensate its effect on the controller. In this case, the concept of torque compensation is similar to the approach presented in this paper, however, the control scheme is based on a PD with a second order derivative controller; therefore, there is no integral action to overcome the steady state error, coming from friction forces or wrongly identifying the parameters of the system. This approach leads to a fast response and a relatively small tracking error when tracking high amplitude references, but fails to produce exact results when tracking small amplitude references; these results can be seen in Fig. 10.

\section{Experimental and simulated validation}

Fig. 11 shows a photograph of the experimental setup utilized to validate the proposed control scheme. The setup consists of (a) a DC motor, Metal Gearmotor 20Dx41L mm 12V CB model from Pololu with the reduction gearbox removed, driven by an ESCON Module 24/2, 4-Q servo controller from Maxon Motor acting as a current controller, (b) a pair of inextensible fishing lines (acting as strings for twisting), Berkley Fireline Svartzonker Braid 0.3 model, aligned along the rotational axis of the motor and attached to the shaft of the motor at one end, and (c) a linear slider connected to the strings, which is attached to an external load that can be changed by adding or removing weight. In addition, three sensors were used: 1) an encoder with a resolution of 20 counts per revolution to measure the motor's angle, 2) a custom-made force sensor integrated into the structure of the TSA device to measure the external axial force applied to

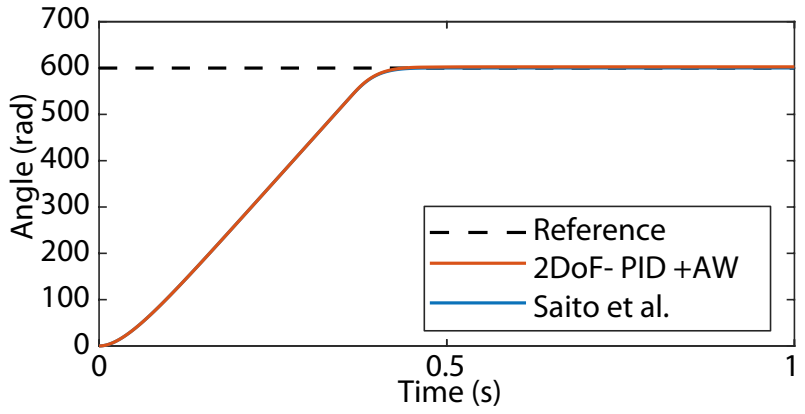

(a)

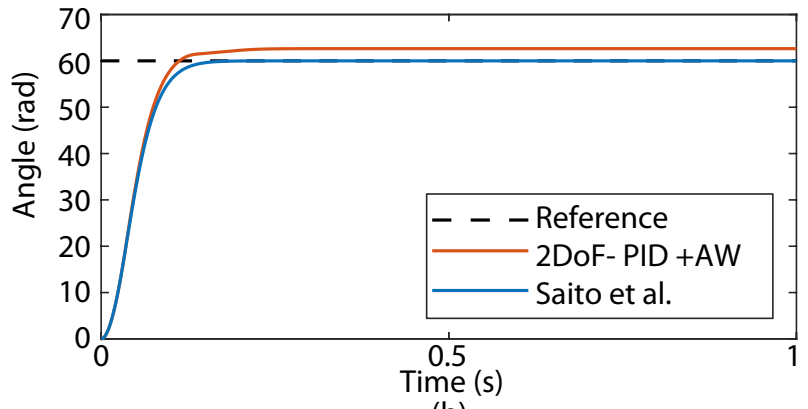

(b)

Figure 10: Closed-loop performance comparison between the control methodology proposed in [31] and our methodology. (a) Example of performance when tracking high amplitude signals. (b) Example of steady state error produced by the lack of integral term of the controller in [31]

the system (more details on the sensor can be found in [9]), and 3) a laser Doppler vibrometer Polytec OFV-5000, equipped with a sensor head OFV-534, to measure the linear motion of the slider. The physical parameters of the system are given in Table 1. The whole control scheme runs on a PC OPTIPLEX 780 with an Intel(R) Core(TM)2 Duo Processor running at $3.167 \mathrm{GHz}$ and equipped with 2 GB of DDR3 RAM memory and a PCI-6621 data acquisition card from National Instruments, which samples and records the data at $1000 \mathrm{~Hz}$. 


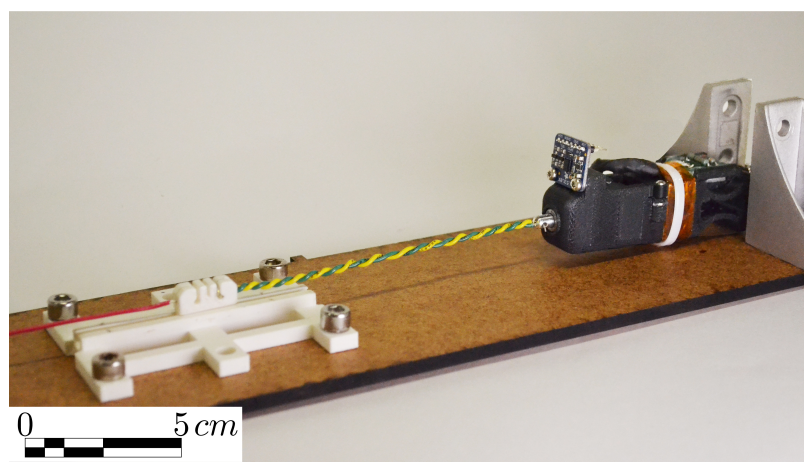

Figure 11: Experimental TSA control platform.

\begin{tabular}{|l|l|l|}
\hline Parameter & Symbol & Value \\
\hline Motor constant & $K_{m}$ & $6.2 \cdot 10^{-}$ \\
Inertia of the motor and hub & $J_{0}$ & $1.67 \cdot 10$ \\
Dynamic friction coefficient & $\nu$ & $4.175 \cdot 1$ \\
Coulomb friction torque & $\Gamma_{p}$ & 0.00217 \\
Length of the strands & $L_{0}$ & 0.195 \\
Strand radius & $r$ & $0.23 \cdot 10$ \\
Number of strands & $\mathrm{n}$ & 2 \\
\hline
\end{tabular}

Table 1: Parameters of the experimental platform

\subsection{Validation of the inner control loop}

The identified transfer-function of the DC motor is as follows:

$$
\frac{\theta(s)}{V_{c}(s)}=M(s)=\frac{3715.2}{s(s+25)}
$$

Based on this, the simulated time response of the system is used to maximize the closed-loop \pm $3 \mathrm{~dB}$ bandwidth of the system for references with amplitudes within the range $[20,100] \cdot 2 \pi$ rads. The combination of the closed-loop poles $p_{1}$ and $p_{2}$ that delivers the best response was found to be $p_{1}=60, p_{2}=50$. The parameters of the controller obtained on applying equations (20), and (34) are shown in the Table 2

\begin{tabular}{|l|l||l|l|}
\hline Parameter & Value & Parameter & Value \\
\hline$g$ & 195 & $T_{i}$ & 0.028 \\
$b_{0}$ & 124.03 & $T_{d}$ & 0.0047 \\
$b_{1}$ & 3.695 & $K$ & 0.45 \\
$N$ & 0.92 & $K_{A W}$ & 2.23 \\
\hline
\end{tabular}

Table 2: Optimal values for the 2-Dof controller

In order to evaluate the effect of the antiwindup action on the closed-loop bandwidth, the closed-1enp system was evaluated when tracking a series of / chirp signals sweeping from $0.1 \mathrm{~Hz}$ to $10 \mathrm{~Hz}$. $\mathrm{H}^{2} \mathrm{~d}$ with amplitudes in the range of $\left[2 \mathrm{O}_{6} 10 \mathrm{Q} \mathrm{m}_{2 \mathrm{~s}}^{2 \pi} \mathrm{rad}\right.$. Taking the reference signal as inpyt to the system and the angular position of the motor as output, the frequency respyonse functions (FRFs) were computed using the Fourier transform of the data. The experimental results and simulated results are shown in Figure 12 for three different scenarios: a) the bandwidh of the motor controlled by a traditional 2-DoF controller without anti-windup action, obtained from simulations (in green), b) the bandwidth produced using the $2-\mathrm{DoF}+\mathrm{AW}$ controller obtained from simulations (in blue), and c) the experimental results of the $2-\mathrm{DoF}+\mathrm{AW}$ controller (red circles).

It is relevant to point out that the dynamic behavior described in (18) (that leads to a theoretical bandwidth of $5.1 \mathrm{~Hz}$ ) is an approximation of the experimental system, which is only fulfilled in the linear range of operation of the motor. It is well-known that when an actuator saturates, the performance of the closed-loop system designed without considering actuator saturation may seriously deteriorate [12]. In this case, the maximum voltage that can be applied to the motor imposes the saturation for the actuator, 


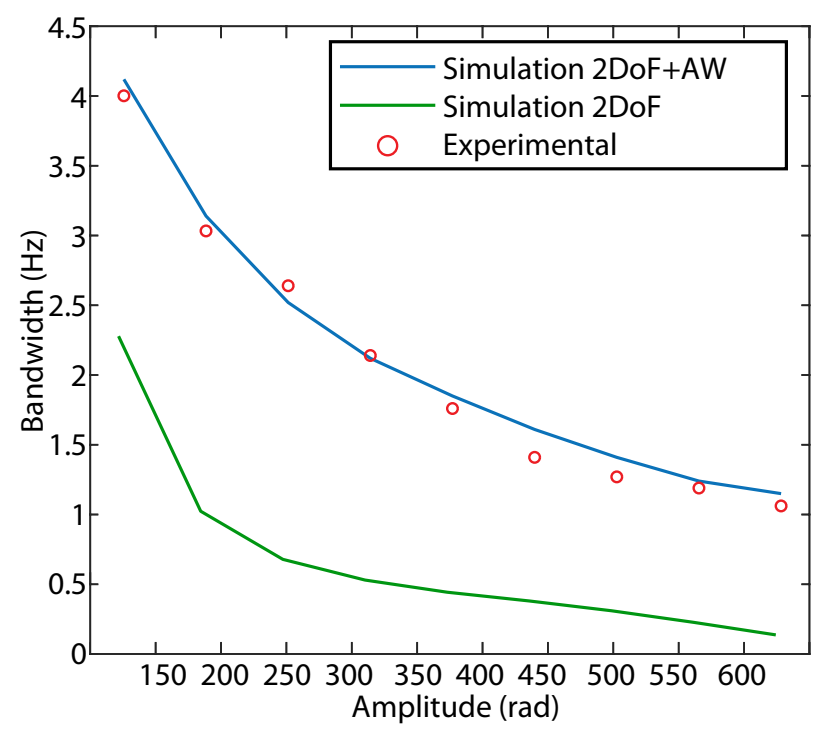

Figure 12: Closed-loop bandwidth of the position controller without external disturbances.

thus limiting the maximum speed of the motor. This is why as the amplitude of the reference increases, the closed-loop bandwidth of the system decreases (in this case the integral action of the controller winds-up and the actuator saturates).

Taking into account the above phenomena, two important facts emerge from the results presented in Fig. 12: a) in both cases, with and without anti-windup action, the maximum achievable bandwidth is smaller than the theoretical bandwidth of $5.1 \mathrm{~Hz}$, in fact, in the case of the 2-DoF $+\mathrm{AW}$ controller the maximum bandwidth is only $77 \%$ of the theoretical maximum. This is because even for amplitudes of $20 \mathrm{rad}$, the actuator saturates and produces a distortion in closed-loop, b) the effect of the ant-windup action is to increase the bandwidth for the whole range of amplitudes; this occurs as despite the actuator still saturating, there is no windup of the integral action. This effect is especially rel- evant in the worst case scenario, i.e. when the reference has the larger amplitude, and the increment of bandwidth compared to the traditional 2 -DoF is of $1100 \%$

\subsection{Validation of the outer control loop}

If we take into account the radius and length of the string given in Table 1, the maximum angle that can be produced by the motor can be computed using (6), leading to 548.3 rads and therefore, to a maximum bandwidth of $1.17 \mathrm{~Hz}$. This maximum bandwidth determines the design of the impedance controller, imposing an upper limit for the natural frequency of the virtual spring given by (23) and equal to $1.17 \mathrm{~Hz}$ or $7.35 \mathrm{rad} / \mathrm{s}$.

In order to validate the design of the impedance controller, two different combinations of mass-spring damper system were chosen, underdamped and critically damped. The parameters of the first case were $k=980 \mathrm{Nm}^{-1}$, a natural frequency of $\omega_{n}=\pi \mathrm{rad} / \mathrm{s}$, and a damping ratio $\zeta=0.2$, while the parameters in the second case were $k=980 \mathrm{Nm}^{-1}, \omega_{n}=2 \pi \mathrm{rad} / \mathrm{s}$, and $\zeta=1$.

Following the definition of $\omega_{n}$ and $\zeta$ as:

$$
\omega_{n}=\sqrt{\frac{k}{m}}, \zeta=\frac{b}{2 \omega_{n} m}
$$

leads to $m=99.29 \mathrm{Kg}$, and $b=124.77 \mathrm{Nsm}^{-1}$ for the first case scenario, and $m=24.82 \mathrm{Kg}$, and $b=$ $311.90 \mathrm{~N} \mathrm{sm}^{-1}$ for the second case. Considering the above values of $k, m$ and $b$, the expression given by (23) was used to provide the impedance controller.

The experimental procedure for testing the performance of the proposed controller consisted of setting the nominal value of the linear position of the end effector $P^{*}$, to a fixed value of 170 
$\mathrm{mm}$ and then suddenly applying a constant force to the end effector, using a known weight and a pulley. In both cases, the maximum weight that the prototype could withstand without breaking was used $(0.7 \mathrm{Kg})$ in order to test the controller in the most demanding situation, i.e. when the external force has the maximum admissible value. Experimental and simulated results for both scenarios are shown in Figs. 13 and 14 respectively.

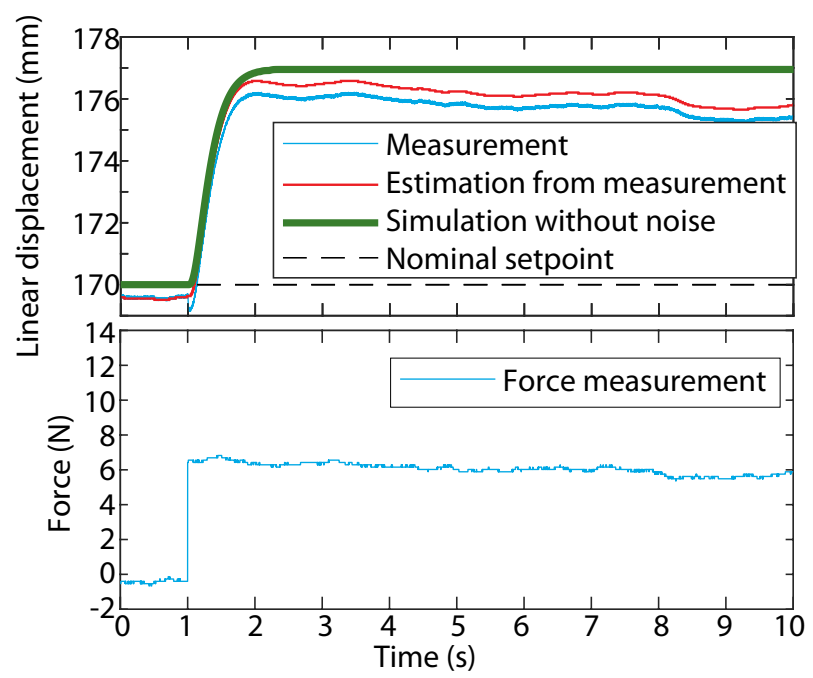

Figure 13: Experimental time response of the linear position of the end effector under addition of external load. The outer loop is designed to mimic an underdamped mass-damper-spring system. Experimental readings of the force sensor are also displayed.

Both figures show the simulated evolution of the end effector considering that force measurements are perfect and without noise (green line in the upper panel graph), and the experimental recorded evolution of the end effector (blue line in the upper panel graph). Additionally, the actual force measurement using the custom sensor of the TSA prototype is shown in blue in

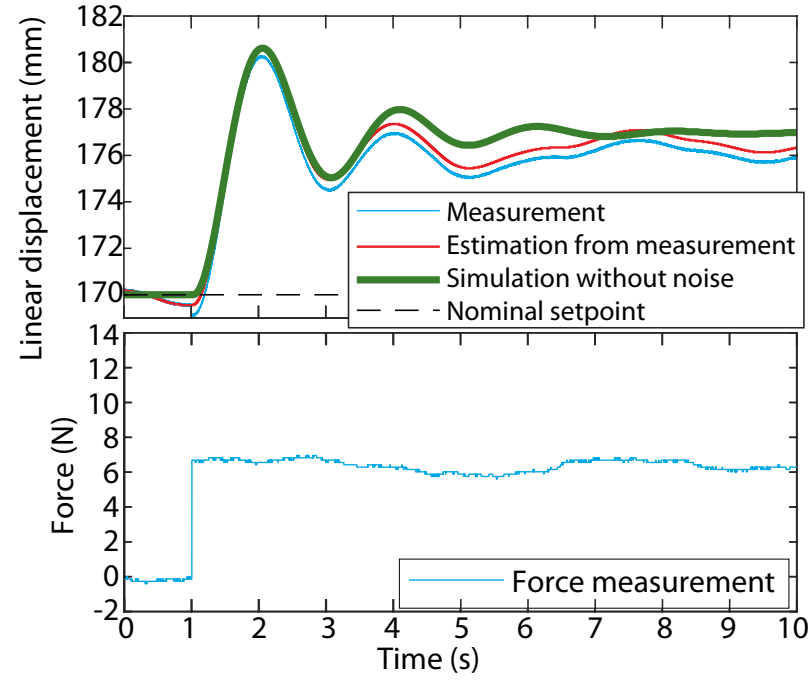

Figure 14: Experimental time response of the linear position of the end effector under addition of an external load. The outer loop is designed to mimic a critically damped mass-damper-spring system. Experimental readings of the force sensor are also displayed.

the lower graph, and the estimation of the linear displacement of the end effector computed from the measurements of force and angular position of the motor (red line in the upper panel graph)

It can be seen that because of the noise in the force measurements, the actual displacement of the end effector is slightly deviated from the simulated prediction, demonstrating that the overall stability of the system only relies on the stability of the inner control loop (which was analyzed and guaranteed in the previous section). In addition, the effect of the noise in the force measurements is due only to the deterioration of the compliance effect of the system. However, this issue can be solved easily by improving the design of the custom force sensor used in the TSA prototype. 
These experiments show that the proposed control scheme is suitable for HMI as the external control loop provides a customizable, compliant mass-spring-damper behavior that allows safe interaction between the user and TSA. As our intended haptic application consists of a mechanical glove in which the TSA produces displacements of the user's fingers, not enough compliance in the interface of the system could potentially harm the user due to the high reduction ratio of the TSA.

\section{Conclusions}

In this paper, we introduce a novel design for the classical 2-Dof controller where addition of a third degree of freedom (in the form of an antiwindup gain) enables us to remove the problem of windup. We analyzed the stability conditions of the traditional 2-Dof controller and its limit conditions, to derive a design rule for the value of the anti-windup gain giving an optimal response. We showed that by torque decoupling the string and motor dynamics, simple rules of linear systems could be used to design the controller, providing good performance, whereby the maximum closed-loop bandwidth could be increased by up to a $1100 \%$ compared to traditional controllers and reaching up to $77 \%$ of the theoretical maximum provided by an equivalent linear system.

The proposed controller has the potential for use in a wide array of functions including control of flexible robots as these systems are already based on 2-Dof controllers and torque decoupling $[6,3]$. The improvements in performance demonstrated in this paper will greatly benefit these applications. Indeed, the innovative controller has already been used as a base to build an impedance controller in which the dy- namic response of the linear position of the end effector of the TSA was designed to mimic the dynamic response of a mass-spring-damper system. In this case, all the parameters (natural frequency, damping ratio and stiffness) can be designed and tailored to the desired goals. Despite the many challenges of controlling TSAs, we are currently using the complete control algorithm as a TSA system in a haptic device under development, where resolution and bandwidth are key requirements to providing realistic sensations to the user.

\section{References}

[1] K. J. AAström and T. Hägglund. PID Controllers: Theory, Design, and Tuning. Instrument Society of America, Research Triangle Park, NC, 2 edition, 1995.

[2] K. Åström and B. Wittenmark. ComputerControlled Systems: Theory and Design, Third Edition. Dover Books on Electrical Engineering. Dover Publications, 2011.

[3] J. C. Cambera and V. Feliu-Batlle. Inputstate feedback linearization control of a single-link flexible robot arm moving under gravity and joint friction. Robotics and Autonomous Systems, 88:24 - 36, 2017.

[4] M. J. Cromie, K. G. Witherspoon, M. Grant, N. I. Kernbaum, R. Mahoney, M. L. Tayson-Frederick, L. C. Fielding, V. Riggs, E. Shahoian, M. E. Hogue, et al. Systems and methods for assistive exosuit system, Mar. 1 2018. US Patent App. $15 / 684,466$.

[5] V. Feliu, E. Pereira, and I. M. Díaz. Passivity-based control of single-link flexi- 
ble manipulators using a linear strain feedback. Mechanism and Machine Theory, 71:191 - 208, 2014.

[6] D. Feliu-Talegon and V. Feliu-Batlle. Improving the position control of a two degrees of freedom robotic sensing antenna using fractional-order controllers. International Journal of Control, 90(6):1256-1281, 2017.

[7] I. Gaponov, D. Popov, and J. Ryu. Twisted string actuation systems: A study of the mathematical model and a comparison of twisted strings. IEEE/ASME Transactions on Mechatronics, 19(4):1331-1342, Aug 2014.

[8] I. Godler, T. Sonoda, and K. Sakurai. Modeling and evaluation of a twist drive actuator for soft robotics. Advanced Robotics, 26(7):765-783, 2012.

[9] M. Hosseini, R. Meattini, G. Palli, and C. Melchiorri. A wearable robotic device based on twisted string actuation for rehabilitation and assistive applications. Journal of Robotics, 2017:1 - 12, 2017.

[10] M. Hosseini, A. Sengül, Y. Pane, J. De Schutter, and H. Bruyninck. Exotenglove: A force-feedback haptic glove based on twisted string actuation system. In 2018 27th IEEE International Symposium on Robot and Human Interactive Communication (RO-MAN), pages 320-327, Aug 2018.

[11] M. Hosseini, A. Sengül, Y. Pane, J. D. Schutter, and H. Bruyninckx. Haptic perception of virtual spring stiffness using exoten-glove. In 2018 11th International
Conference on Human System Interaction (HSI), pages 526-531, July 2018.

[12] T. Hu and Z. Lin. Control Systems with Actuator Saturation: Analysis and Design. Control Engineering. Birkhäuser Boston, 2001.

[13] L. Hua, X. Sheng, and X. Zhu. Highperformance transmission mechanism for robotic applications. Mechanism and $\mathrm{Ma}$ chine Theory, 105:176 - 184, 2016.

[14] S. H. Jeong and K. Kim. A 2-speed small transmission mechanism based on twisted string actuation and a dog clutch. IEEE Robotics and Automation Letters, 3(3):1338-1345, July 2018.

[15] S. H. Jeong, K. Kim, and S. Kim. Designing anthropomorphic robot hand with active dual-mode twisted string actuation mechanism and tiny tension sensors. IEEE Robotics and Automation Letters, 2(3):1571-1578, July 2017.

[16] H. Lee, H. Choi, and S. Park. Accurate modeling and nonlinearity compensation in the speed mode of a twisted string actuator. Mechanism and Machine Theory, 137:53 66, 2019.

[17] J. Lin and R. Chu. A novel measurement model of twisted string actuating system. In 2018 International Automatic Control Conference (CACS), pages 1-6, Nov 2018.

[18] U. Mehmood, D. Popov, I. Gaponov, and J. Ryu. Rotational twisted string actuator with linearized output: Mathematical model and experimental evaluation. In 2015 IEEE International Conference on 
Advanced Intelligent Mechatronics (AIM), pages 1072-1077, July 2015.

[19] K. S. Narendra and J. H. Taylor. Frequency domain criteria for absolute stability. Academic Press, 1973.

[20] S. Nedelchev, I. Gaponov, and J. Ryu. Design of robotic gripper with constant transmission ratio based on twisted string actuator: Concept and evaluation. In 2018 IEEE/RSJ International Conference on Intelligent Robots and Systems (IROS), pages 967-972, Oct 2018.

[21] K. Ogata. Modern Control Engineering. Instrumentation and controls series. Prentice Hall, 2010.

[22] G. Palli, C. Melchiorri, G. Vassura, U. Scarcia, L. Moriello, G. Berselli, A. Cavallo, G. D. Maria, C. Natale, S. Pirozzi, C. May, F. Ficuciello, and B. Siciliano. The dexmart hand: Mechatronic design and experimental evaluation of synergy-based control for human-like grasping. The International Journal of Robotics Research, 33(5):799824, 2014.

[23] G. Palli, C. Natale, C. May, C. Melchiorri, and T. Wurtz. Modeling and control of the twisted string actuation system. IEEE/ASME Transactions on Mechatronics, 18(2):664-673, April 2013.

[24] I. Park and V. SunSpiral. Impedance controlled twisted string actuators for tensegrity robots. In 201414 th International Conference on Control, Automation and Systems (ICCAS 2014), pages 1331-1338, Oct 2014.
[25] J. Park, J. H. Kim, K. Kim, and S. Kim. Design and control of antagonistic robot joint with twisted string actuators. In 2016 13th International Conference on Ubiquitous Robots and Ambient Intelligence (URAI), pages 563-565, Aug 2016.

[26] A. Pepe, M. Hosseini, U. Scarcia, G. Palli, and C. Melchiorri. Development of an haptic interface based on twisted string actuators. In 2017 IEEE International Conference on Advanced Intelligent Mechatronics (AIM), pages 28-33, July 2017.

[27] D. Popov, I. Gaponov, and J. Ryu. Bidirectional elbow exoskeleton based on twistedstring actuators. In 2013 IEEE/RSJ International Conference on Intelligent Robots and Systems, pages 5853-5858, Nov 2013.

[28] V. M. Popov. Absolute stability of nonlinear systems of automatic control. Automation and Remote Control, 22(8):857-875, 1962.

[29] K. S. Rattan, V. M. R. Feliũ, and H. B. Brown. A robust control scheme for flexible arms with friction in the joints. In $N A S A$ Air Force Workshop Space Operations Automation and Robotics, Dayton, OH July, 1988.

[30] L. Rundqwist. Anti-reset windup for pid controllers. IFAC Proceedings Volumes, 23(8, Part 4):453 - 458, 1990. 11th IFAC World Congress on Automatic Control, Tallinn, 1990 - Volume 4, Tallinn, Finland.

[31] Y. Saito, U. Nishio, T. Nozaki, and K. Ohnishi. Acceleration-based position and force control for twist drive. In 2013 
IEEE International Conference on Mechatronics (ICM), pages 664-669, Feb 2013.

[32] A. San-Millan, V. Feliu-Batlle, and S. S. Aphale. Two-degrees-of-freedom pi2d controller for precise nanopositioning in the presence of hardware-induced constant time delay. ISA Transactions, 2019.

[33] U. Scarcia, L. Moriello, A. Pepe, G. Palli, and C. Melchiorri. Design of a twistedstring actuator for haptic force rendering. IFAC-PapersOnLine, 51(22):479 - 485, 2018. 12th IFAC Symposium on Robot Control SYROCO 2018.

[34] Y. J. Shin, H. J. Lee, K. Kim, and S. Kim. A robot finger design using a dual-mode twisting mechanism to achieve high-speed motion and large grasping force. IEEE Transactions on Robotics, 28(6):1398-1405, Dec 2012 .

[35] H. Singh, D. Popov, I. Gaponov, and J.-H. Ryu. Twisted string-based passively variable transmission: Concept, model, and evaluation. Mechanism and Machine Theory, 100:205 - 221, 2016.

[36] B. Suthar, M. Usman, H. Seong, I. Gaponov, and J. Ryu. Preliminary study of twisted string actuation through a conduit toward soft and wearable actuation. In 2018 IEEE International Conference on Robotics and Automation (ICRA), pages 2260-2265, May 2018.

[37] M. Suzuki and A. Ichikawa. Toward springy robot walk using strand-muscle actuators. In Climbing and Walking Robots, pages 479-486, Berlin, Heidelberg, 2005. Springer Berlin Heidelberg.
[38] S. Uozumi, K. Yu, N. Yoshimura, and K. Ohnishi. Motion control of tendondriven rotary actuator using twist drive system. In 2014 IEEE 13th International Workshop on Advanced Motion Control (AMC), pages 470-475, March 2014.

[39] Q. Xu. Robust impedance control of a compliant microgripper for high-speed position/force regulation. IEEE Transactions on Industrial Electronics, 62(2):1201-1209, 2015 .

[40] Yo-Sung Cho and K. Narendra. An offaxis circle criterion for stability of feedback systems with a monotonic nonlinearity. IEEE Transactions on Automatic Control, 13(4):413-416, August 1968. 